\title{
Cucumber mosaic virus-Plant Interactions: Identification of 3a Protein Sequences Affecting Infectivity, Cell-to-Cell Movement, and Long-distance Movement
}

\author{
Qiubo Li, ${ }^{1}$ Ki Hyun Ryu, ${ }^{1}$ and Peter Palukaitis ${ }^{1,2}$ \\ ${ }^{1}$ Department of Plant Pathology, Cornell University, Ithaca, NY 14853, U.S.A.; ${ }^{2}$ Pathology Division, Scottish \\ Crop Research Institute, Invergowrie, Dundee DD2 5DA, U.K. \\ Accepted 11 November 2000.
}

\begin{abstract}
Mutants of the Cucumber mosaic virus (CMV) movement protein (MP) were generated and analyzed for their effects on virus movement and pathogenicity in vivo. Similar to the wild-type MP, mutants M1, M2, and M3, promoted virus movement in eight plant species. Mutant M3 showed some differences in pathogenicity in one host species. Mutant M8 showed some host-specific alterations in movement in two hypersensitive hosts of CMV. Mutant M9 showed altered pathogenicity on three hosts and was temperature sensitive for long-distance movement, demonstrating that cell-to-cell and long-distance movement are distinct movement functions for CMV. Four mutants (M4, M5, M6, and M7) were debilitated from movement in all hosts tested. Mutants M4, M5, and M6 could be complemented in trans by the wild-type MP expressed transgenically, although not by each other or by mutant M9 (at the restrictive temperature). Mutant M7 showed an inability to be complemented in trans. From these mutants, different aspects of the CMV movement process could be defined and specific roles for particular sequence domains assigned. The broader implications of these functions are discussed.
\end{abstract}

\footnotetext{
The movement of most plant viruses within their hosts involves several distinct stages: translocation of a recently replicated virus within the inoculated cell to the intercellular connections and the plasmodesmata, trafficking through the plasmodesmata and through adjacent cells to bundle sheath cells, movement into and through different cell types of the vascular bundles from bundle sheath cells through vascular parenchyma cells and companion cells to the sieve elements, movement in the phloem through the sieve tube elements, exit into young companion cells of newly developing leaves, and translocation through cells of vascular bundles to the mesophyll and epidermal cells of the systemically infected tissues (Carrington et al. 1996; Lazarowitz and Beachy 1999; Lucas
}

Corresponding author. P. Palukaitis; Telephone +44 1382568523 ; Fax: +44 1382 562426. E-mail: ppaluk@scri.sari.ac.uk

Current address of K. H. Ryu: Department of Horticultural Science, Seoul Woman's University, Seoul 138-774, Korea. and Gilbertson 1994; Reichel et al. 1999). The research focused on movement within and between cells, with plants being assessed for various cellular barriers to virus movement (Carrington et al. 1996; Lazarowitz and Beachy 1999; Nelson and Van Bel 1998). These barriers probably reflect the major form of resistance to virus infection: the inhibition of virus movement (Dawson and Hilf 1992; Gilbertson and Lucas 1996; Meshi and Okada 1987). The interactions between virus movement proteins (MPs) and the cell ultrastructure to promote movement within the cell (e.g., across the nuclear membrane or along the cytoskeleton within cells) and via plasmodesmata or tubules formed between cells have opened up new areas of research in plant virology, cell biology, and cell physiology (Ding et al. 1999; Lazarowitz and Beachy 1999; Lucas and Wolf 1999; Reichel et al. 1999). MPs may have evolved to either coopt or mimic native plant proteins or processes involved in translocation within and between plant cells (Gilbertson and Lucas 1996). It also has been suggested that plant virus MPs may have been this type of plant protein at one stage (Lucas and Gilbertson 1994; Lucas and Wolf 1999).

Depending on the virus system, plant viruses encode one to five proteins involved in their movement (Carrington et al. 1996; Lazarowitz and Beachy 1999; Maule 1991). For some viruses, the coat protein(s) (CP) of the virus as well as the $\mathrm{MP}(\mathrm{s})$ are involved in virus movement. In other viruses, there is not just one protein required for virus movement but a complex of three proteins encoded by overlapping genes, referred to as the "triple gene block." In some cases, the movement of such viruses also may require CP (Carrington et al. 1996; Lazarowitz and Beachy 1999; Nelson and Van Bel 1998). For some nuclear-associated viruses, one MP has the role of translocation from the nucleus to the cytoplasm, whereas another functions in gating between cells (Lazarowitz and Beachy 1999). In other cases, the MP is associated with tubules that form between cells and contain isometric virus particles (Carrington et al. 1996; Lazarowitz and Beachy 1999; Maule 1991). In spite of the seeming multiplicity of forms of movement, many MPs show similar characteristics whether or not they share the same requirements for cell-to-cell (local) and long-distance (systemic) movement (Carrington et al. 1996; Lazarowitz and Beachy 1999; Melcher 2000; Mushegian and Koonin 1993). In addition, for some viruses gene products 
involved in virus replication appear to be involved in virus movement (Deom et al. 1997; Gal-On et al. 1994; Nelson and Van Bel 1998). Models for the movement of viral genomes from the site of replication (or assembly) to adjacent cells have been proposed (Carrington et al. 1996; Gilbertson and Lucas 1996; Lazarowitz and Beachy 1999), but there are still many unanswered questions, including why some viruses seem to have elements of different movement mechanisms and what is the nature of the molecular basis of the host specificity of virus movement.

To begin addressing some of these issues, we mutated the gene encoding the MP of Cucumber mosaic virus (CMV) to identify functional domains of the movement process. CMV occurs worldwide as biologically differentiable isolates, called strains. The collective host range of these strains is approximately 1,241 species in 101 plant families (Edwardson and Christie 1991). CMV contains five genes expressed from three genomic and two subgenomic RNAs. All five encoded proteins appear to have some effect on virus movement. This includes the two replication-associated proteins (98 and 111 $\mathrm{kDa})$, the viral CP $(25 \mathrm{kDa})$, a protein involved in suppressing plant responses $(13 \mathrm{kDa})$, and the $3 \mathrm{a}$ protein $(30 \mathrm{kDa})$ (Ding et al. 1995a; Ding et al. 1995b; Kaplan et al. 1995; Palukaitis et al. 1992). The 3a protein shares several of the above features common to a number of MPs and thus is referred to as the CMV MP (Canto et al. 1997; Ding et al. 1995a; Kaplan et al. 1995; Li and Palukaitis 1996). We constructed a series of alanine-scanning mutants in the 3a gene of the Fny strain of CMV with procedures first used to mutate the MP of the dianthovirus Red clover necrotic mosaic virus and delineate functional domains of this MP (Fujiwara et al. 1993; Giesman-Cookmeyer and Lommel 1993). The CMV 3a MP mutants were then assessed for their affects on CMV, vis-à-vis infectivity and pathogenicity on eight host species, and the ability of the mutants to be complemented in trans by CMV MP expressed in transgenic plants. A functional map of the CMV MP is presented, incorporating the effects of various mutations and alterations described here and previously. Ad- ditional novel interactions suggested by the data presented here also are discussed.

\section{RESULTS}

Target selection for alanine scanning mutagenesis.

A series of seven alanine-scanning mutants (designated M1 to M7) initially was created by mutating adjacent or nearby pairs of codons to alanine within the CMV 3a protein. These mutations were made at sequences corresponding to 3 a protein positions R8 plus T9 (M1), E38 plus D40 (M2), Y75 plus D76 (M3), R97 plus T98 (M4), Y144 plus D145 (M5), R156 plus F158 (M6), and N191 plus Y192 (M7) where at least one of each pair was a charged or polar residue. The mutation of such paired sequences reduced the likelihood of reversion. These sequences were selected on the basis of being in regions highly conserved between strains of CMV and three bromoviruses (Fig. 1), where sequences involved in common functions are more likely to be located. Two other mutants were then created in areas of less sequence similarity. Because mutant M8 (D20 and D21) is located in a region proximal to the $\mathrm{N}$ terminus where CMV and bromoviruses showed considerable sequence divergence, it was thought that this region would be more likely to affect specific rather than generic interactions. Mutant M9 (P60) involved a highly conserved proline that potentially could affect the folding of the MP. No mutations were made beyond position 192 (M7) for reasons discussed below. In addition, there is virtually no sequence similarity between CMV and the bromoviruses beyond position 209 (Fig. 1).

\section{Infectivity and accumulation of alanine-scanning mutants in tobacco plants.}

The nine MP mutants were assessed initially for their infectivity in tobacco. RNA 3 transcribed from the mutant cDNA clones of pFny309 was combined with CMV RNAs 1 and 2 transcribed from wild-type cDNA clones pFny109 and pFny209, and the RNAs were inoculated in tobacco. These

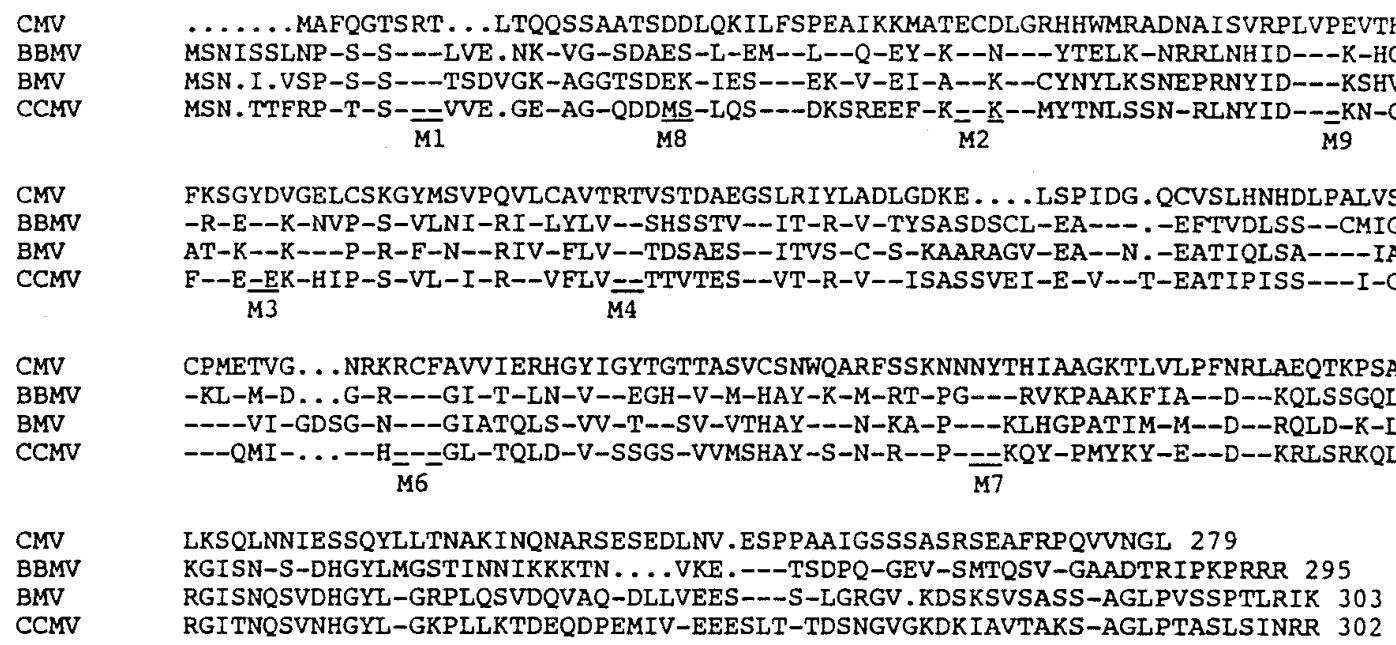

Fig. 1. Comparison of the amino acid sequences of the movement proteins (MPs) of Cucumber mosaic virus (CMV) and the three bromoviruses: Brome mosaic virus (BMV), Broadbean mottle virus (BBMV), and Cowpea chlorotic mottle virus (CCMV). Dashes indicate sequence identity of bromovirus MP sequences to that of the CMV MP. Dots indicate the absence of corresponding amino acids in the sequence. Amino acid sequence s altered in the nine alanine-scanning mutants (M1 to M9) are underlined. 
RNAs containing MP mutants M1, M2, M3, M8, and M9 (referred to below by the mutant number) were able to systemically infect tobacco plants (Table 1). All but mutant M9 showed a similar time course of symptom development and produced comparable yields of virus ( 0.23 to $0.28 \mathrm{mg}$ of virus per $g$ of leaves), somewhat less than what was obtained for wild-type Fny-CMV derived from infectious RNA transcripts (0.35 mg per g) (Table 1). Mutant M9 produced mild mosaic and vein-clearing symptoms on tobacco versus the severe mosaic and leaf distortion typical of wild-type Fny-CMV. The yield of virus ( $0.21 \mathrm{mg}$ per $\mathrm{g}$ ), however, was comparable to the yields for the other mutants described above.

In contrast, mutants M4, M5, M6, or M7 did not induce any symptoms on tobacco and no accumulating viral RNA could be detected by Northern blot hybridization of total RNAs extracted from either the inoculated or upper leaves (Fig. 2A and data not shown). Mutants M4, M5, and M6, however, were able to infect transgenic tobacco plants expressing the wildtype Fny-CMV 3a protein (Table 1 and Fig. 2B). The yield of virus was less than that observed for the other mutants $(0.13$, 0.18 , and $0.17 \mathrm{mg}$ per $\mathrm{g}$ for M4, M5, and M6, respectively). The apparent variation in accumulation of the various CMV RNAs visible in Figure 2 was not observed consistently in other experiments (data not presented). The symptoms elicited by mutants M4, M5, and M6 were limited to the vein-clearing previously observed with 3 a deletion mutants (Kaplan et al. 1995). Except for some plants infected by mutant M4, virus purified from these 3a-transgenic tobacco was unable to infect nontransformed tobacco (data not presented), indicating that neither had the mutations reverted nor had the recombination that occurred with the wild-type $3 a$ gene in the transgenic plants. The altered regions of the progeny virus RNAs were sequenced, and the sequence was as expected for each mutant (data not presented).

The various mutants M4, M5, M6, and M7 also were coinoculated in six pairwise combinations to nontransgenic tobacco plants. In no case was infection detected by symptoms or Northern blot hybridization of RNA extracted from the inoculated leaves (data not presented). This indicates that the mutants were unable to complement each other.

\section{Accumulation of alanine-scanning mutants in tobacco protoplasts.}

Mutant M7 was unable to infect either nontransformed or 3a-transgenic tobacco (Table 1 and Fig. 2). Because the 3a

Table 1. Infectivity and complementation of Cucumber mosaic virus movement protein mutants in non- and 3a-transgenic tobacco

\begin{tabular}{llc}
\hline Inoculum & Tobacco & 3a-Tobacco $^{\text {a }}$ \\
\hline WT $^{\mathrm{b}}$ & $+^{\mathrm{c}} ;(0.35)^{\mathrm{d}}$ & + \\
M1 (R8, T9) & $+;(0.28)$ & + \\
M2 (E38, D40) & $+;(0.23)$ & + \\
M3 (Y75, D76) & $+;(0.27)$ & + \\
M4 (R97, T98) & - & $+;(0.13)$ \\
M5 (Y144, D145) & - & $+;(0.18)$ \\
M6 (R156, F158) & - & $+;(0.17)$ \\
M7 (N191, Y192) & - & - \\
M8 (D20, D21) & $+;(0.24)$ & + \\
M9 (P60) & $+;(0.21)$ & + \\
\hline
\end{tabular}

a Transgenic tobacco expressing the 3 a movement protein.

b $\mathrm{WT}=$ wild-type $3 \mathrm{a}$ movement protein.

${ }^{c}$ Local and systemic infection (+) versus no detectable infection (-).

${ }^{d}$ Yield of virus in milligrams of virus per gram of tissue. gene is dispensable for the replication of CMV RNA 3, it did not seem likely that the RNA mutation in mutant M7 had a direct, cis-acting effect on the ability of RNA 3 to be replicated. It was possible, however, that the encoded 3 a protein in mutant M7 had a cis- or trans-acting effect on the replication of the CMV RNAs. To examine this possibility, protoplasts were prepared from nontransformed and 3a-transgenic tobacco and transfected with transcripts containing either the wild-type RNA 3 or the various MP mutants. The RNAs extracted from the protoplasts were analyzed $24 \mathrm{~h}$ postinoculation by Northern blot hybridization (Fig. 3A). Here it is apparent that all three genomic CMV RNAs were able to replicate and accumulate for mutant M7 to levels similar to that of the wild-type virus or the other MP mutants. In addition, there was no spe-

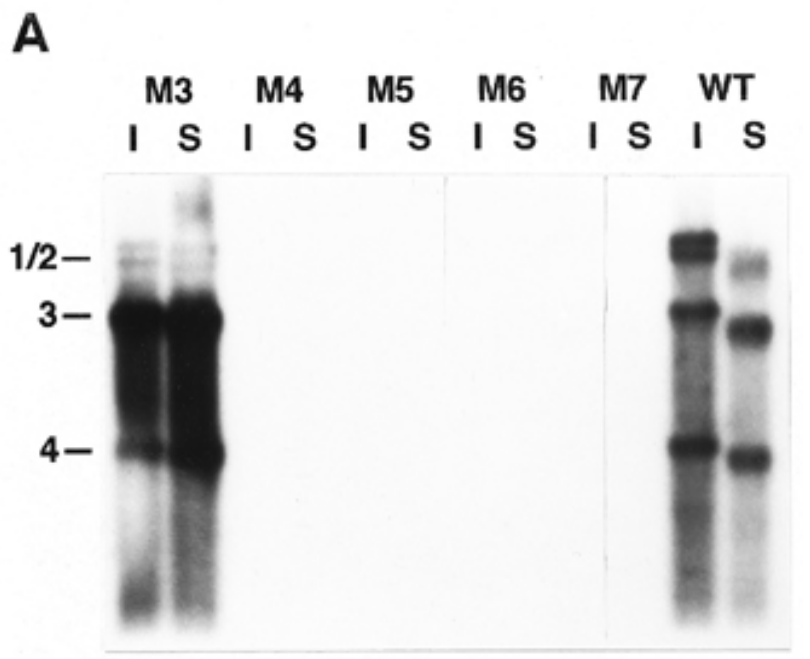

B

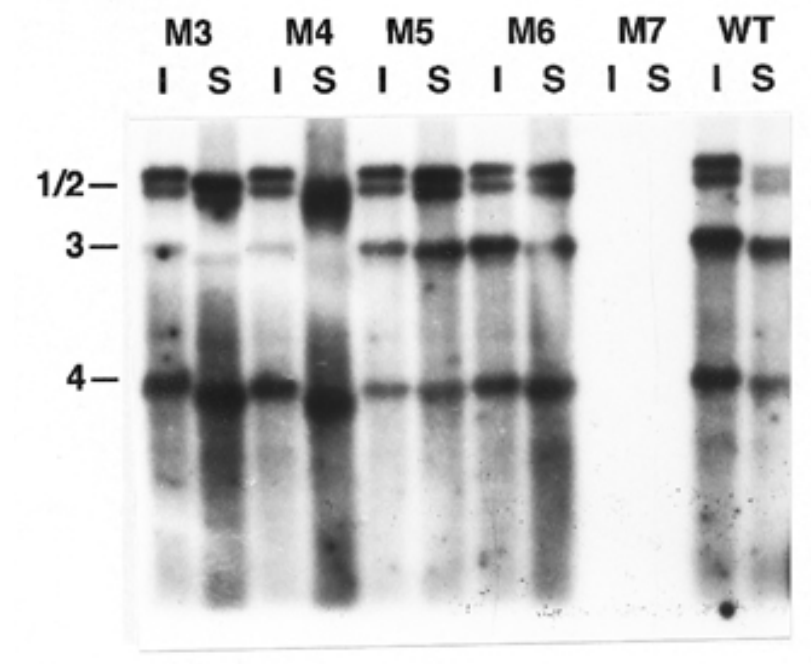

Fig. 2. Cucumber mosaic virus (CMV) accumulation in plants infected by wild-type (WT) CMV and the alanine-scanning mutants (M3-M7). Samples were obtained from inoculated plants: A, nontransformed tobacco plants; B, 3a-transgenic tobacco plants. RNAs were extracted from inoculated leaves (I) or leaves above the inoculated leaves (S) and analyzed by Northern blot hybridization with a probe specific to the $3^{\prime}$ common nontranslated region of each viral RNA and the subgenomic RNA (4). The positions of the CMV RNAs 1, 2, 3, and 4 are indicated. 
cific effect on the generation of the subgenomic RNA 4 encoding the CP gene for mutant M7 versus the other mutants. In the absence of CMV CP, CMV RNAs accumulate poorly or not noticeably in protoplasts (Canto et al. 1997; Suzuki et al. 1991). The expression and accumulation of the MP and CP in protoplasts for mutant M7 were similar to those of the other MP mutants in tobacco protoplasts (Fig. 3B). Thus, it is unlikely that the mutant M7 3a protein is less stable than the wild-type $3 \mathrm{a}$ protein. Moreover, any hypothetical reduced stability would not account for the inability of mutant M7 to be complemented by the transgenic 3 a protein (Table 1 and Fig. 2B). In fact, the defective 3 a protein of mutant M7 is cis dominant for movement of CMV RNA 3 and possibly trans dominant for movement of CMV RNAs 1 and 2 in the 3atransgenic plants.

\section{Infectivity of alanine-scanning mutants in other hosts.}

Table 2 shows the results of infectivity analyses of the nine mutants on other CMV hosts, including plants in the families Solanaceae (tomato and pepper), Cucurbitaceae (cucumber and squash), Compositae (Zinnia elegans), Chenopodiaceae (Chenopodium quinoa), and Leguminosae (cowpea). In all of the systemic host species (excluding the hypersensitive hosts $C$. quinoa and cowpea in which Fny-CMV induces local lesions), those mutants that could infect tobacco also could in-

\section{A}

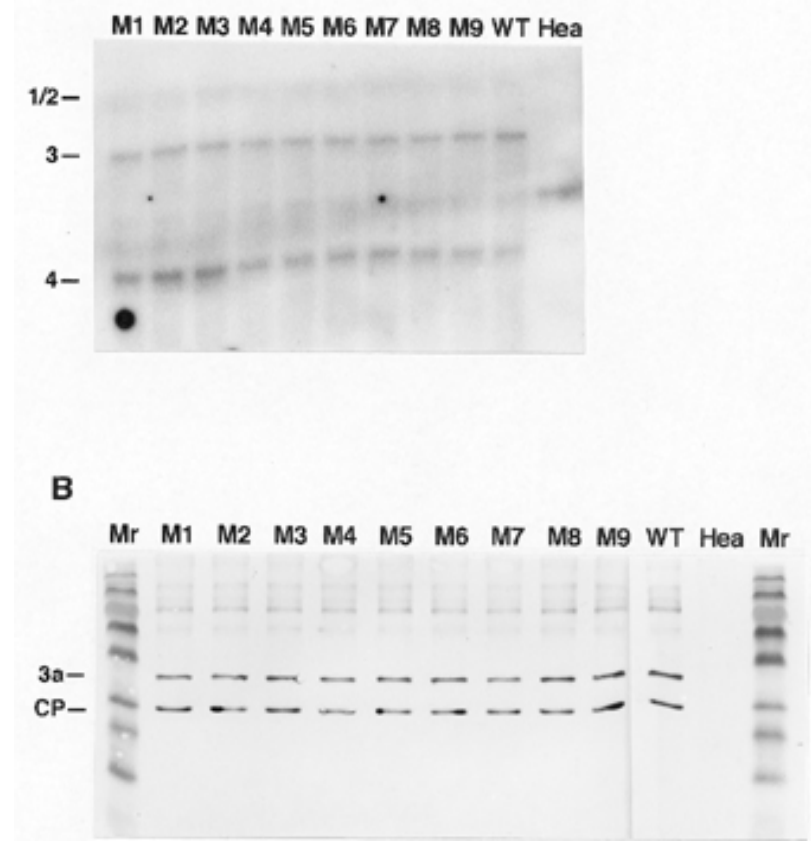

Fig. 3. Cucumber mosaic virus (CMV) accumulation in tobacco protoplasts. Wildtype (WT) CMV and the nine alanine-scanning mutants were transfected into tobacco protoplasts. At $24 \mathrm{~h}$ posttransfection, A, total RNAs and B, proteins were extracted and analyzed separately. The RNAs were analyzed by Northern blot hybridization with a probe specific to the $3^{\prime}$ end of each viral RNA $(\mathbf{A})$. Proteins were analyzed by Western blotting and immunoprobing sequentially with antisera to the 3a MP and the CP, respectively, before photography $(\mathbf{B})$. Hea = extracts from noninoculated protoplasts. Position of the CMV RNAs 1, 2, 3, and 4 (A) and the $3 \mathrm{a}$ MP and $\mathrm{CP}$ are indicated $(\mathbf{B}) . \mathrm{Mr}=$ protein molecular weight markers. fect the other host species (compare Tables 1 and 2). None of the mutants defective for movement on tobacco (M4 to M7) could infect any of the other CMV hosts, indicating that the effects of those mutations were on the more generic functions of the MP rather than on its host-specific functions.

In tomato, mutant M9 induced more severe symptoms than the wild-type CMV, but the level of accumulated viral RNA for mutant M9 was similar to that of the wild-type CMV (data not shown). The symptoms induced by mutants M9 and M3 were milder than they were for the other mutants or for wildtype CMV in Z. elegans, also presumably reflecting some difference in host-specific interactions. Most strikingly, however, was the effect of the mutation in the 3 a gene of mutant M8 on the infection of the two hypersensitive hosts, $C$. quinoa and cowpea (Table 2). In both of these species, mutant M8 did not induce a hypersensitive response and no viral RNA could be detected in the inoculated leaves by Northern blot hybridization (data not shown). Further experiments testing mutant M8 on C. amaranticolor showed that mutant M8 was unable to move out of the initially inoculated epidermal cells. Moreover, the requirement for cell-to-cell movement rather than just the coexpression of MP and CP is essential for the elicitation of a hypersensitive response in this host (Canto and Palukaitis 1999a).

Effects of mutations on long-distance movement.

Mutants M1 to M3, M8, and M9 were assessed for potential temperature-sensitive (ts) effects on virus movement in to-

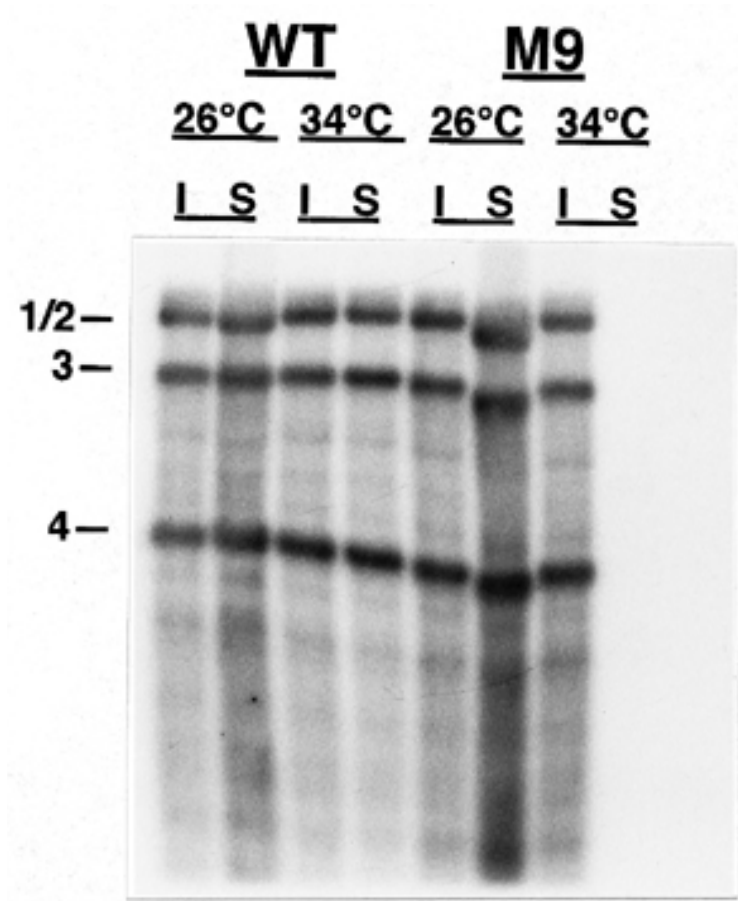

Fig. 4. Effect of temperature on Cucumber mosaic virus (CMV) accumulation in tobacco plants. Wild-type (WT) CMV and CMV containing movement protein mutant M9 were inoculated to tobacco plants maintained for 7 days at 26 or $34^{\circ} \mathrm{C}$. Total RNAs were extracted from inoculated leaves (I) as well as leaves above the inoculated leaves (S) and analyzed by Northern blot hybridization with a CMV-specific probe. Positions of the four CMV RNAs are indicated. Increased mobility of RNAs in the sample from leaves infected systemically (S) by mutant M9 and maintained at $26^{\circ} \mathrm{C}$ is an electrophoretic artifact. 
bacco. Mutant M9 showed a ts effect on virus movement at $34^{\circ} \mathrm{C}$ (Fig. 4), whereas the other mutants did not (data not presented). Below $32^{\circ} \mathrm{C}$, however, mutant M9 did not show a ts effect on virus movement (Fig. 4 and data not presented). Mutant M9 could still move cell to cell in the inoculated leaf at $34^{\circ} \mathrm{C}$ but could not move long distance (Fig. 4). In contrast, wild-type Fny-CMV could move long distance at $34^{\circ} \mathrm{C}$ (Fig. 4). After shifting the temperature from 34 to $26^{\circ} \mathrm{C}$, mutant $\mathrm{M} 9$ could again move long distance (systemically) in tobacco that was initially inoculated and maintained at $34^{\circ} \mathrm{C}$ (not shown). This confirms previous experiments that indicate a role for the CMV MP in long-distance and cell-to-cell movement (Kaplan et al. 1997; Ryabov et al. 1999).

Mutants M4, M5, and M6 were each mixed separately with mutant M9 and inoculated to tobacco plants that were maintained at $34^{\circ} \mathrm{C}$. No complementation for long-distance movement was observed between the mutants affecting cell-to-cell movement (M4, M5, and M6) and the mutant M9, which affected systemic infection of tobacco (data not shown).

\section{DISCUSSION}

\section{Functional map of the CMV MP.}

A functional map of the CMV 3a MP can be constructed (Fig. 5) based on data presented here and elsewhere (Canto and Palukaitis 1999a; Canto and Palukaitis 1999c; Canto et al. 1997; Ding et al. 1995a; Gal-On et al. 1996; Kaplan et al. 1995; Kaplan et al. 1997; Li and Palukaitis 1996; Vaquero et al. 1997; You et al. 1999). Although this map, similar to those proposed for the functional maps of Tobacco mosaic virus (TMV) (Kahn et al. 1998; Mezitt and Lucas 1996), Cauliflower mosaic virus (Huang et al. 2000; Thomas and Maule 1999), Cowpea mosaic virus (Bertens et al. 2000), and Red clover necrotic mosaic virus (Mezitt and Lucas 1996), is linear, it is recognized that the three-dimensional structure of the MP is likely to be important for functionality, at the very least through coordination of the independently analyzed functions. Superimposed on this map of sites affecting various functions (Fig. 5) are several putative functional domains and conserved or variable sequences that are based on sequence analyses (De
Jong et al. 1995; Fujita et al. 1996; Gafny et al. 1992; Nagano et al. 1997; Romero et al. 1992). Thus, it can be seen that all of the mutants that exhibited an inhibition of virus movement are clustered in the center of the MP, whereas two conditionally dysfunctional mutants (M8 and M9) are grouped in the Nterminal region at amino acids 20 and 21 and 60. At position 51 is another site associated with a conditional dysfunction (S3), an inability to systemically infect cucurbit species (Kaplan et al. 1997). Position 51 is equivalent to BMV MP position 54 (Fig. 1), and mutations at this site are associated with a change in host range of BMV (De Jong et al. 1995). The sequences from amino acids 213 to 279 (the $\mathrm{C}$ terminus) showed virtually no similarity between CMV and the bromoviruses, whereas the MPs of CMV strains themselves showed considerable variation between amino acids 228 and 279 (Fig. 1 and data not shown). In addition, CMV MP sequences from position 237 to 279 could be deleted without affecting movement, whereas some or all of amino acids 209 to 236 were required for some aspect of the movement process (Kaplan et al. 1995). The sequences between amino acids 174 to 233 were shown to contain the core of the in vitro RNA binding domain (Vaquero et al. 1997). This domain includes mutant M7 (amino acids 191 and 192) as well as sequences required for movement (amino acids 209 to 236) (Kaplan et al. 1995). The deletion of amino acids 160 to 200 or 200 to 246 , however, did not affect RNA binding in vitro (You et al. 1999). This may indicate the presence of more than one RNA binding site available within this region. While the C-terminal 40 amino acids of the CMV MP appeared to be dispensable, amino acid 240, located in this region, together with amino acid 51 conditioned for a cucurbit-specific, restrictedmovement phenotype (Kaplan et al. 1997) and for increased spread within tobacco plants (Gal-On et al. 1996). Therefore, the $\mathrm{C}$ terminus of the 3 a protein appears to contain sequences that have subtle effects on host-specific interactions (Nagano et al. 1997). This C-terminal region of the MP also appears to be involved in interactions involving the CP (Nagano et al. 1997).

The N-terminal region of the MP also contains sequences involved in host-specific interactions, as shown by mutant M8

Table 2. Infectivity of Cucumber mosaic virus (CMV) movement protein mutants in other host plant species

\begin{tabular}{|c|c|c|c|c|c|c|c|}
\hline \multirow[b]{2}{*}{ Inoculum } & \multicolumn{7}{|c|}{ Host plants ${ }^{a}$} \\
\hline & $\begin{array}{l}\text { Lycopersicon } \\
\text { esculentum }\end{array}$ & Capsicum annum & Cucumis sativus & Cucurbita реро & $\begin{array}{l}\text { Zinnia ele- } \\
\text { gans }\end{array}$ & $\begin{array}{c}\text { Chenopodium } \\
\text { quinoa }\end{array}$ & $\begin{array}{l}\text { Vigna un- } \\
\text { guiculata }\end{array}$ \\
\hline $\mathrm{WT}^{\mathrm{b}}$ & + & + & $t^{\mathrm{d}}$ & + & +++ & $L L^{f}$ & LL \\
\hline M1 (R8, T9) & + & + & + & + & +++ & LL & LL \\
\hline M2 (E38, D40) & + & + & + & + & +++ & LL & LL \\
\hline M3 (Y75, D76) & + & + & + & + & $++^{\mathrm{e}}$ & LL & LL \\
\hline M4 (R97, T98) & - & - & - & - & - & - & - \\
\hline M5 (Y144, D145) & - & - & - & - & - & - & - \\
\hline M6 (R156, F158) & - & - & - & - & - & - & - \\
\hline M7 (N191, Y192) & - & - & - & - & - & - & - \\
\hline M8 (D20, D21) & + & + & + & + & +++ & - & - \\
\hline M9 (P60) & $++^{c}$ & + & + & + & $++^{\mathrm{e}}$ & LL & LL \\
\hline
\end{tabular}

${ }^{a}$ Host plants tested for infectivity: L. esculentum (tomato), C. annum (pepper), C. sativus (cucumber), C. pepo (squash), Z. elegans, C. quinoa, V. unguiculata (cowpea).

${ }^{\mathrm{b}} \mathrm{WT}=$ wild-type $3 \mathrm{a}$ movement protein.

${ }^{c}$ Symptoms more severe than for WT CMV.

${ }^{\mathrm{d}}$ Local and systemic infection (+) versus no detectable infection (-).

e Symptoms of mutants M3 and M9 on Z. elegans were much milder than with WT CMV.

${ }^{\mathrm{f}} \mathrm{LL}=$ local lesions with no systemic infection. 
(Table 2). Deletion of the N-terminal 34 amino acids was shown to lead to a loss of virus movement, whereas mutations at amino acid 2, 3, 8, and 9 did not affect movement (Kaplan et al. 1995). Similarly, duplications of amino acid 4 and 5, generated in vivo by pseudoreversion of a frameshifted mutant, led to the production of a virus that appeared to move normally (Kaplan et al. 1995). Thus, in contrast to the situation with TMV (Gafny et al. 1992), the sequence of the Nterminal nine amino acids of the CMV MP does not appear to be critical for movement, whereas deletion of the first 34 amino acids affected either the stability or function of the MP (Kaplan et al. 1995; You et al. 1999).

Dysfunctional mutant M4 is located within a hydrophobic core domain (amino acids 86 to 108) conserved in putative MPs of 17 plant virus genera (Melcher 2000; Mushegian and Koonin 1993). This hydrophobic domain contains a putative transmembrane helix, which may be conserved in cellular transport proteins. In contrast, dysfunctional mutants M5, M6, and $\mathrm{M} 7$ are located in sequence blocks that resemble putative zinc fingers and possible RNA binding domains (Fig. 5). Within these putative zinc finger regions are putative nucleic acid binding domains previously identified in bromoviruses (Romero et al. 1992). MP mutant M5 is at the end of the first putative zinc finger region (Fig. 5). and was shown to be unable to traffic itself or viral RNA from cell to cell or to increase the size exclusion of fluorescent dextran (Ding et al. 1995a). The sequences in the second putative nucleic acid binding domain of BMV, equivalent to CMV amino acid 165 that is near mutant M6 (amino acids 156 and 158), was altered in a bromovirus hybrid that developed the ability to systemically infect cowpeas (Fijita et al. 1996). Thus, this region of the MP contains sequences affecting generic functions of the MP as well as host-specific functions.

\section{Complementation of movement functions: models for virus movement.}

The inability of mutant M7 to be complemented in trans by the transgenic 3a protein suggests that this mutant MP is inter- fering with the movement of CMV. This particular mutation in RNA 3 did not affect its replication nor did the mutated MP interfere with the replication and accumulation of any of the other genomic RNAs or the expression of the subgenomic RNA required for CP production (Fig. 3). Rather, the effects of the mutation appeared to be on some aspect(s) of the movement process, per se. In this regard, mutant M7 behaved similar to the transdominant mutations made in the $\mathrm{N}$-terminal region of the TMV 30-kDa MP (Lapidot et al. 1993) and within the 26-kDa MP of the potexvirus White clover mosaic virus (Beck et al. 1994). In those cases, transgenic expression of the mutant MPs led to resistance to infection by the homologous wild-type virus as well as provided some level of protection against infection by a range of distantly related and unrelated viruses (Beck et al. 1994; Cooper et al. 1995; Lapidot et al. 1993). It is of interest to determine whether mutant M7 could also exhibit such broad protection to other viruses if expressed in transgenic plants. The mechanism of such protection and the ability of mutant M7 to inhibit movement could provide valuable insight into the mechanisms of virus movement.

Complementation of CMV movement by infection with four CMV RNAs (RNA 1, RNA 2, and two dysfunctional forms of RNA 3) has been demonstrated (Canto and Palukaitis 1999a; Canto and Palukaitis 1999b; Canto and Palukaitis 1999c; Canto et al. 1997). In those instances, however, complementation involved dysfunction in different genes (MP and $\mathrm{CP}$ ). The inability of mutants to complement each other here may be understandable for mutants M4 to M6, which are in close proximity and may affect the same or overlapping functional domains. Although mutant M9 could move cell to cell at the restrictive temperature (Fig. 4), the inability of mutants M4 to M6 to complement mutant M9 at the restrictive temperature could indicate that mutants M4 to M6 also have an effect on long-distance movement or that a single-movement protein must be able to interact with the viral RNA and various host components.

Mutant M8 was capable of systemic infection in the various systemic hosts tested (Tables 1 and 2). Moreover, MP

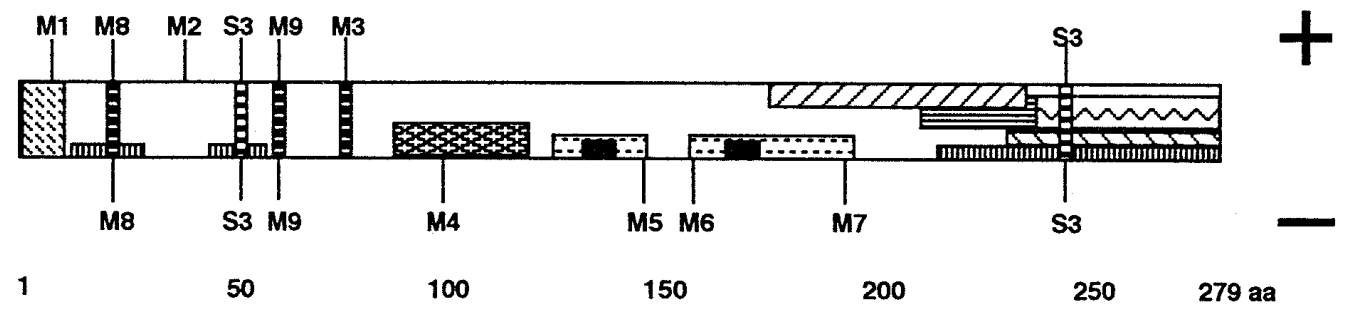

Fig. 5. Mutational and functional map of the Cucumber mosaic virus (CMV) 3a movement protein (MP). Positions of MP mutants M1-M9 and S3 are indicated. Filled-pattern rectangles indicate various domains or conserved regions, described below. Plus sign indicates MP infectious mutants, whereas minus sign indicates noninfectious MP mutants. Conditionally infectious MP mutants (M8, M9, and S3) are indicated. MP S3 indicates two positions (51 and 240) that together influence movement in tobacco and cucurbit species. MP mutant M1 (positions 8 and 9) lies within an N-terminal region (amino acids 1-9) where deletion and duplication mutants do not affect CMV movement. MP mutant M2 (positions 38 and 40) lies between two regions (10-25 and 44-57) of strong sequence dissimilarity between the MPs of CMV and the bromoviruses (Fig. 1). MP mutant M8 (positions 20 and 21 ) lies within one of these regions. MP mutants M9 (position 60) and M3 (positions 75 and 76) lie between the above sequence-heterogeneous regions and a highly conserved hydrophobic core (positions 86-118) conserved in the putative MPs of 17 plant virus genera. MP mutant M4 lies within this hydrophobic core region. MP mutants M5 (positions 144 and 145), M6 (positions 156 and 158), and M7 (positions 191 and 192) lie within two putative zinc finger domains (positions 126-146 and 157-194), which also encompass two putative nucleic acid binding domains (black rectangles at positions 134-138 and 164-168). MP mutant M7 also is located within the in vitro RNA binding domain (diagonal pattern fill at positions 174-233). Domain bordered by amino acid positions 209-236 (horizontal-line pattern fill) overlaps the RNA binding domain and contains sequences essential for CMV movement. Adjacent domain bordered by amino acids $237-279$ (C terminus) is dispensable for CMV movement. Sequences in this region also show the greatest variability between CMV and the bromoviruses (vertical-line pattern fill at positions 213-279) or between strains of CMV (diagonal-line pattern fill at positions 228-279). 
mutant M8 was able to associate with the plasmodesmata of epidermal cells (Canto et al. 1999a), yet it was not able to promote movement of RNA or MP to other epidermal cells (Canto et al. 1999c). CMV containing mutant M8, however, was able to move systemically in most host species tested (Tables 1 and 2). Thus, MP mutant M8 must retain the ability to bind and interact with viral RNA. This indicates that there are viral-encoded, active processes that occur in CMV movement beyond the association of the RNA:MP complex with plasmodesmata. This could involve a conformational change in the MP, as previously proposed for the role of the CP in cell-to-cell movement (Ryabov et al. 1999), or it could involve plasmodesmata-associated proteins that differ slightly between epidermal-epidermal and epidermalmesophyll cells.

A model has been proposed for the movement of CMV RNA into sieve elements (Blackman et al. 1998). This model predicts that CMV RNA and MP and CP interact to form a ribonucleoprotein complex that moves through the plasmodesmata between companion cells and the sieve element. Various data suggest that CMV RNA does not move cell to cell in the form of virus particles even though $\mathrm{CP}$ is required for CMV movement (Blackman et al. 1998; Kaplan et al. 1998; Schmitz and Rao 1998). Rather, the data suggest that RNA must be associated with MP for cell-to-cell movement and CP plays an important, but possibly indirect, role. No interaction has been detected directly between MP and CP (O'Reilly et al. 1997; D. Szilassy and P. Palukaitis unpublished). Because replication proteins (Deom et al. 1997; GalOn et al. 1994; Nelson and Van Bel 1998) and the CMV 2b protein (Ding et al. 1995b) also affect virus movement, it is of interest to determine whether they also participate directly in the long-distance movement process.

\section{MATERIALS AND METHODS}

\section{Viral constructs, alanine-scanning mutagenesis, transcription, and plant inoculation.}

Plasmids pFny109, pFny209, and pFny309 contain fulllength cDNA copies of RNA 1, RNA 2, and RNA 3, respectively, of the Fny strain of CMV (Rizzo and Palukaitis 1990). Nine alanine-scanning mutations were generated in the $3 \mathrm{a}$ gene of Fny-CMV by site-directed mutagenesis (Kunkel et al. 1987) with the use of synthetic oligonucleotide primers. The mutated DNA was digested with restriction enzymes and subcloned into pFny309 by standard procedures (Sambrook et al. 1989). The nucleotide changes were then confirmed by DNA sequencing. The plasmids pFny109, pFny 209, pFny309, and various derivatives of the last were transcribed in vitro with T7 RNA polymerase, and the viral RNA transcripts were inoculated to various plant species, as previously described (Zhang et al. 1994). The plant species inoculated were tobacco (Nicotiana tabacum cv. Xanthi nc), tomato (Lycopersicon esculentum cv. Rutgers), pepper (Capsicum annum cv. Jalapeño), cucumber (Cucumis sativus cv. Straight 8), zucchini squash (Cucurbita pepo cv. Black Beauty), C. quinoa, cowpea (Vigna unguiculata cv. Blackeye 5), and Z. elegans. Transgenic tobacco plants expressing the CMV 3a gene and competent for complementation of CMV defective for movement (Kaplan et al. 1995) also were used in some experiments.

\section{Analysis of viral RNAs and viral-encoded proteins.}

Tobacco protoplasts were prepared and transfected with CMV RNA transcripts as previously described (Gal-On et al. 1994; Gal-On et al. 1995). Proteins were extracted from infected protoplasts and were analyzed by electroblotting and immunoprobing proteins with antisera to the $3 \mathrm{a}$ and $\mathrm{CP}$ (sequentially). RNAs were extracted from infected protoplasts and analyzed by Northern blot hybridization with a probe specific to the $3^{\prime}$ nontranslated and common to each of the four CMV RNAs. Protein and RNA levels were equalized prior to loading gels. All of the above were done as previously described by Gal-On et al. (1994). Total RNAs were extracted from either inoculated leaves or leaves above the inoculated leaves and analyzed for the presence of the CMV RNAs by Northern blot hybridization (Gal-On et al. 1994; Gal-On et al. 1995).

\section{ACKNOWLEDGMENTS}

This work was supported in part by grant DE-FG02-86ER13505 from the U.S. Department of Energy and by a grant-in-aid (SCR/462/96) from the Scottish Executive Rural Affairs Department.

\section{LITERATURE CITED}

Beck, D. L., Guildford, P. J., Voot, D. M., Andersen, M. T., and Forster, R. L. S. 1991. Triple gene block proteins of white clover mosaic potexvirus are required for transport. Virology 183:695-702.

Bertens, P., Wellink, J., Goldbach, R., and Van Kammen, A. 2000. Mutational analysis of the cowpea mosaic virus movement protein. Virology 267:199-208.

Blackman, L. M., Boevink, P., Santa Cruz, S., Palukaitis, P., and Oparka, K. J. 1998. The movement protein of cucumber mosaic virus traffics into sieve elements in minor veins of Nicotiana clevelandii. Plant Cell 10:525-537.

Canto, T., and Palukaitis, P. 1999a. The hypersensitive response to cucumber mosaic virus in Chenopodium amaranticolor requires virus movement outside the initially infected cell. Virology 265:74-82.

Canto, T., and Palukaitis, P. 1999b. Replicase-mediated resistance to cucumber mosaic virus does not inhibit localization and/or trafficking of the viral movement protein. Mol. Plant-Microbe Interact. 12:743747.

Canto, T., and Palukaitis, P. 1999c. Are tubules generated by the 3a protein necessary for cucumber mosaic virus movement? Mol. PlantMicrobe Interact. 12:985-993.

Canto, T., Prior, D. A. M., Hellwald, K.-H., Oparka, K. J., and Palukaitis, P. 1997. Characterization of cucumber mosaic virus. IV. Movement protein and coat protein are both essential for cell-to-cell movement of CMV. Virology 237:237-248.

Carrington, J. C., Kasschau, K. D., Mahajan, S. K., and Schaad, M. C. 1996. Cell-to-cell and long-distance transport of viruses in plants. Plant Cell 8:1669-1681.

Cooper, B., Lapidot, M., Heick, J. A., Dodds, J. A., and Beachy, R. N. 1995. A defective movement protein of TMV in transgenic plants confers resistance to multiple viruses whereas the functional analog increases susceptibility. Virology 206:307-313.

Dawson, W. O., and Hilf, M. E. 1992. Host-range determinants of plant viruses. Annu. Rev. Plant Physiol. Plant Mol. Biol. 43:527-557.

De Jong, W., Chu, A., and Ahlquist, P. 1995. Coding changes in the 3a cell-to-cell movement gene can extend the host range of brome mosaic virus systemic infection. Virology 214:646-674.

Deom, C. M., Quan, S., and He, X. Z. 1997. Replicase proteins as determinants of phloem-dependent long-distance movement of tobamoviruses in tobacco. Protoplasma 199:1-8.

Ding, B., Li, Q., Nguyen, L., Palukaitis, P., and Lucas, W. J. 1995a. Cucumber mosaic virus 3 a protein potentiates cell-to-cell trafficking of CMV RNA in tobacco plants. Virology 207:345-353.

Ding, S.-W., Li, W.-X., and Symons, R. H. 1995b. A novel naturally occurring hybrid gene encoded by a plant RNA virus facilitates long 
distance virus movement. EMBO J. 14:5762-5772.

Ding, B., Itaya, A., and Woo, Y.-M. 1999. Plasmodesmata and cell-tocell communication in plants. Intl. Rev. Cytol. 190:251-316.

Edwardson, J. R., and Christie, R. G. 1991. Cucumoviruses. Pages 293 319 in: CRC Handbook of Viruses Infecting Legumes. J. R. Edwardson and R. G. Christie, eds. CRC Press, Boca Raton, FL, U.S.A.

Fujita, Y., Mise, K., Okuno, T., Ahlquist, P., and Furusawa, I. 1996. A single codon change in a conserved motif of a bromovirus movement protein gene confers compatibility with a new host. Virology 223:283-291.

Fujiwara, T., Giesman-Cookmeyer, D., Ding, B., Lommel, S. A., and Lucas, W. J. 1993. Cell-to-cell trafficking of macromolecules through plasmodesmata potentiated by the red clover necrotic mosaic virus movement protein. Plant Cell 5:1783-1794.

Gafny, R., Lapidot, M., Berna, A., Holt, C. A., Deom, C. M., and Beach, R. N. 1992. Effects of terminal deletion mutations on function of the movement protein of tobacco mosaic virus. Virology 187:499-507.

Gal-On, A., Kaplan, I., Roossinck, M. J., and Palukaitis, P. 1994. The kinetics of infection of zucchini squash by cucumber mosaic virus indicate a function for RNA 1 in virus movement. Virology 205:280289.

Gal-On, A., Kaplan, I., and Palukaitis, P. 1995. Differential effects of satellite RNA on the accumulation of cucumber mosaic virus RNAs and their encoded protein in tobacco vs. zucchini squash with two strains of CMV helper virus. Virology 208:58-68.

Gal-On, A., Kaplan, I. B., and Palukaitis, P. 1996. Characterization of cucumber mosaic virus. II. Identification of movement protein sequences that influence its accumulation and systemic infection in tobacco. Virology 226:354-361.

Giesman-Cookmeyer, D., and Lommel, S. A. 1993. Alanine scanning mutagenesis of a plant virus movement protein identifies three functional domains. Plant Cell 5:973-982.

Gilbertson, R. L., and Lucas, W. J. 1996. How do viruses traffic on the "vascular highway?" Trends Plant Sci. 1:260-267.

Huang, Z., Han, Y., and Howell, S. H. 2000. Formation of surface tubules and fluorescent foci in Arabidopsis thaliana protoplasts expressing a fusion between the green fluorescent protein and cauliflower mosaic virus movement protein. Virology 271:58-64.

Kahn, T. W., Lapidot, M., Heinlein, M., Reichel, C., Cooper, B., Gafney, R., and Beachy, R. N. 1998. Domains of the TMV movement protein involved in subcellular localization. Plant J. 15:15-25.

Kaplan, I. B., Shintaku, M. H., Li, Q., Zhang, L., Marsh, L. E., and Palukaitis, P. 1995. Complementation of virus movement in transgenic tobacco expressing the cucumber mosaic virus 3 a gene. Virology 209:188-199.

Kaplan, I. B., Gal-On, A., and Palukaitis, P. 1997. Characterization of cucumber mosaic virus. III. Localization of sequences in the movement protein controlling systemic infection in cucurbits. Virology 230:343-349.

Kaplan, I. B., Zhang, L., and Palukaitis, P. 1998. Characterization of cucumber mosaic virus. V. Cell-to-cell movement requires capsid protein but not virions. Virology 246:221-231.

Kunkel, T. A., Roberts, J. D., and Zakous, K. A. 1987. Rapid and efficient site-directed mutagenesis without phenotypic selection. Methods Enzymol. 155:367-382.

Lapidot, M., Gafny, R., Ding, B., Wolf, S., Lucas, W. J., and Beachy, R. N. 1993. A dysfunctional movement protein of tobacco mosaic virus that partially modifies the plasmodesmata and limits spread in transgenic plants. Plant J. 4:959-970.

Lazarowitz, S., and Beachy, R. N. 1999. Viral movement proteins as probes for intracellular and intercellular trafficking in plants. Plant Cell 11:535-548

Li, Q., and Palukaitis, P. 1996. Comparison of the nucleic acid- and NTP-binding properties of the movement protein of cucumber mosaic cucumovirus and tobacco mosaic tobamovirus. Virology 216:71-79.
Lucas, W. J., and Gilbertson, R. L. 1994. Plasmodesmata in relation to viral movement within leaf tissues. Annu. Rev. Phytopathol. 32:387411.

Lucas, W. J., and Wolf, S. 1999. Connections between virus movement, macromolecular signaling and assimilate allocation. Curr. Opin. Plant Biol. 2:192-197.

Maule, A. 1991. Virus movement in infected plants. Crit. Rev. Plant Sci. 9:457-473.

Melcher, U. 2000. The " $30 \mathrm{~K}$ " superfamily of viral movement proteins. J. Gen. Virol. 81:257-266.

Meshi, T., and Okada, Y. 1987. Systemic movement of viruses. Pages 285-304 in: Plant-Microbe Interactions: Molecular and Genetic Perspectives, Vol. 2. T. Kosuge, and E. W. Nester, ed. Macmillan Publishing Co., New York.

Mezitt, L. A., and Lucas, W. J. 1996. Plasmodesmal cell-to-cell transport of proteins and nucleic acids. Plant Mol. Biol. 32:251-273.

Mushegian, A. R., and Koonin, E. V. 1993. Cell-to-cell movement of plants viruses. Insights from amino acid sequence comparisons of movement proteins and from analogies with cellular transport systems. Arch. Virol. 133:239-257.

Nagano, H., Okuno, T., Mise, K., and Furusawa, I. 1997. Deletion of the C-terminal 33 amino acids of cucumber mosaic virus movement protein enables a chimeric brome mosaic virus to move from cell-to-cell. J. Virol. 71:2270-2276.

Nelson, R. S, and Van Bel, A. J. E. 1998. The mystery of virus trafficking into, through and out of vascular tissue. Prog. Bot. 59:476533.

O'Reilly, E. K., Paul, J. D., and Kao, C. C. 1997. Analysis of the interaction of viral RNA replication proteins by using the yeast two-hybrid assay. J. Virol. 71:7526-7532.

Palukaitis, P., Roossinck, M. J., Dietzgen, R. G., and Francki, R. I. B. 1992. Cucumber mosaic virus. Adv. Virus Res. 41:281-348.

Reichel, C., Mas, P., and Beachy, R. N. 1999. The role of the ER and cytoskeleton in plant viral trafficking. Trends Plant Sci. 4:458-462.

Rizzo, T. M., and Palukaitis, P. 1990. Construction of full-length cDNA clones of cucumber mosaic virus RNAs 1, 2 and 3: Generation of infectious transcripts. Mol. Gen. Genet. 222:249-256.

Romero, J., Dzianott, A. M., and Bujarski, J. J. 1992. The nucleotide sequence and genome organization of the RNA 2 and RNA 3 segments in broad bean mottle virus. Virology 187:671-681.

Ryabov, E. V., Roberts, I. M., Palukaitis, P., and Taliansky, M. 1999. Host-specific cell-to-cell and long-distance movements of cucumber mosaic virus are facilitated by the movement protein of groundnut rosette virus. Virology 260:98-108

Sambrook, J., Fritsch, E. F., and Maniatis, T. 1989. Molecular Cloning: A Laboratory Manual, 2nd ed. Cold Spring Harbor Laboratory Press, Cold Spring Harbor, NY, U.S.A.

Schmitz, I., and Rao, A. L. N. 1998. Deletions in the conserved aminoterminal basic arm of cucumber mosaic virus coat protein disrupt virion assembly but do not abolish infectivity and cell-to-cell movement. Virology 248:323-331.

Suzuki, M., Kuwata, S., Kataoka, J., Masuta, C., Nitta, N., and Takanami, Y. 1991. Functional analysis of deletion mutants of cucumber mosaic virus RNA 3 using an in vitro transcription system. Virology 183:106-113.

Thomas, C. L., and Maule, A. J. 1999. Identification of inhibitory mutants of Cauliflower mosaic virus movement protein function after expression in insect cells. J. Virol. 73:7886-7890.

Vaquero, C., Liao, Y.-C., Nähring, J., and Fischer, R. 1997. Mapping of the RNA-binding domain of the cucumber mosaic virus movement protein. J. Gen. Virol. 78: 2095-2099.

You, J.-S., Baik, H.-S., and Paek, K.-H. 1999. Mutational analysis of cucumber mosaic virus movement protein gene. J. Biochem. Mol Biol. 32:82-85.

Zhang, L., Hanada, K., and Palukaitis, P. 1994. Mapping local and systemic symptom determinants of cucumber mosaic cucumovirus in tobacco. J. Gen. Virol. 75: 3185-3191. 